\title{
Role of MR Defecography in the Assessment of Obstructed Defecation Syndrome
}

\author{
MOHAMED F. OSMAN, M.D.* and AHMED F. AHMED, M.D.** \\ The Departments of Diagnostic \& Interventional Radiology* and General Surgery**, Faculty of Medicine, Cairo University
}

\begin{abstract}
Background: Obstructed Defecation Syndrome (ODS) is characterized by the urge to defecate but an impaired ability to expel the fecal bolus. Symptoms include unsuccessful fecal evacuation attempts, excessive straining, pain, bleeding after defecation, and a sense of incomplete fecal evacuation. ODS has been linked to anatomic abnormalities of rectocele, rectoanal or recto-rectal intussusception, paradoxical puborectalis contraction, pelvic organ prolapse, descending perineum syndrome, solitary rectal ulcer syndrome, sigmoidocele, and enterocele. A functional abnormality such as pelvic floor dyssynergia, decreased rectal compliance and decreased rectal sensation has also been shown to contribute to symptoms of ODS.
\end{abstract}

Objective: To assess the role of MR defecography in finding functional abnormalities in patients with obstructed defecation syndrome.

Patients and Methods: A prospective study was carried out on 30 patients (20 females and 10 males) with clinical symptoms of obstructed defecation syndrome for a period longer than 6 months. Patients were assessed statically using T2 weighted images and dynamically by MR defecography using fast imaging via steady-state acquisition sequences. All patients had symptoms of obstructed defecation syndrome as unsuccessful fecal evacuation attempts, excessive straining, pain during defecation, a sense of incomplete fecal evacuation and digital rectal evacuation.

Results: A total of 30 patients with a mean age of 40.2 with $20(66.7 \%)$ females and $10(33.3 \%)$ males. MR defecography revealed rectocele in $19(63.3 \%)$ of all patients and cystocele in $17(56.7 \%)$ patients. Four patients $(13.3 \%)$ had rectal intussusception. Spastic pelvic floor syndrome was found in 2 patients $(6.75 \%)$. Multicompartmental dysfunction was observed in $18(60 \%)$ of the female patients.

Conclusion: MR defecography was proven to be highly valuable in assessing patients with obstructed defecation syndrome, particularly females as it depicts multicompartmental dysfunction in females with a seemingly single compartment symptom ultimately changing the treatment approach.

Correspondence to: Dr. Mohamed F. Osman, The Department of Diagnostic \& Interventional Radiology, Faculty of Medicine, Cairo University
Key Words: Obstructed defecation syndrome - MR defecography - Rectal prolapse-Pelvic organ prolapse.

\section{Introduction}

OBSTRUCTED defecation syndrome (or simply outlet obstruction) is a subtype of constipation considered as one of the major pelvic floor dysfunctions irritating our ageing population, particularly women over 50 seeking medical care [1]

In concordance with the National Institute for Health and Clinical Excellence (NICE) guidelines published in 2010, Obstructed Defecation Syndrome (ODS) is clinically diagnosed in patients with excessive straining, sense of incomplete fecal evacuation from the rectum, failed evacuation attempts, pelvic heaviness and self-digitations [2]

Assessment of the condition should not be based on physical and clinical examination solely as this would underestimate the compartments involved and the degree of prolapse, particularly in women where the pelvic floor should be dealt with as a single entity. A patient could be complaining of obstructed defecation symptoms denoting posterior compartment dysfunction yet associated disorders in anterior or middle compartments could be detected radiologically necessitating a different scheme of management to avoid postoperative recurrence of symptoms [3]

ODS has been linked to several abnormalities that could be detected radiologically by trans-anal or trans-vaginal ultrasound, dynamic perineal ultrasound, conventional defecography and MR Defecography (MRD). These abnormalities include rectocele, recto-anal or recto-rectal intussusception, rectal prolapse, puborectalis dyskinesia, pelvic organ prolapse, solitary rectal ulcer syndrome, peritonocele and enterocele [4] . 
MRD offers comprehensive evaluation of the underlying anorectal dysfunction by imaging patients at rest and during evacuation, with no ionizing radiation exposure, which is particularly useful as ODS is more common among females in their reproductive age than males. It also provides accurate information on concomitant pelvic floor disorders guiding the surgeon to the appropriate management whether surgical or medical [5].

\section{Patients and Methods}

A prospective study was carried out from December 2016 to June 2017 at the Radiology Department of Kasr Al-Ainy Hospital, Cairo University. Thirty patients (20 females and 10 males) presenting with Obstructed Defecation Syndrome (ODS) symptoms (based on NICE guidelines 2010) were referred to our department and underwent Magnetic Resonance Defecography (MRD). Patients with tumors of the rectum and anal canal, previous pelvic floor surgeries and contraindications to undergo MRI examinations as patients with pacemakers, etc were excluded. Approval from the Ethics Committee at the Radiology Department at Cairo University was obtained and informed consent was taken from all patients.

\section{Equipment:}

MRD was performed in the supine position using a 1.5 Tesla MR Imaging Unit in closed configuration; Acheiva (Philips Medical Systems, Best, the Netherlands) with a pelvic phased-array coil wrapped around the patient.

\section{Techniques and imaging protocol:}

No oral or intravenous contrast agent was administered. Patients were asked to void 2 hours before the examination. Due to the particular nature of the examination, thorough explanation of the technique and training of the patients was done prior to entering the MRI Unit by a doctor to ensure the patient understands how to perform Valsalva maneuver properly. A doctor also attended all the examinations and accompanied the patients in the MRI room during the dynamic scan to make sure they are compliant and to explain what they are expected to do during each phase of the examination.

The rectum was opacified with $300 \mathrm{ml}$ of ultrasound gel while the patient is lying in the left lateral position with the right knee flexed on the scanner table. Pads were placed underneath the patients to protect the coils and preventing them from soiling themselves and ensuring their comfort.
Elevating patients' legs by a pillow was done to facilitate evacuation.

Static T2 weighted images were acquired first in three planes angled on the anal canal; axial, coronal and sagittal, providing anatomic understanding of the anal sphincter and the pelvic floor muscles [repetition time ms/echo time ms (TR/TE) 5000/132, Field of View (FOV) 240-260mm, slice thickness 2-4mm, gap 0-0.5 mm, number of signals acquired 2, flip angle 90, matrix 512 X 512, acquisition time $3.12 \mathrm{~min}$ for each sequence].

Evacuation phase (MRD) was acquired next in the sagittal plane using Balanced Fast Echo (BFFE) sequence (TR/TE 5.0/1.6ms, FOV 300mm, 5 dynamic scans, 7 slices, slice thickness $3.0 \mathrm{~mm}$, gap $0.0 \mathrm{~mm}$ ). The evacuation sequence was repeated until the injected gel is seen passing through the anal canal lumen.

Additional dynamic images were acquired after the evacuation phase in three planes; axial, coronal, and sagittal using Balanced Fast Echo (BFFE) sequence (TR/TE 5.0/1.6ms, FOV 300mm, slice thickness $5-7 \mathrm{~mm}$, gap 0.0mm). Patients were imaged in 5 phases: At rest, during the contraction of pelvic floor (squeezing), during mild straining, during moderate straining and during maximum straining.

Acquired dynamic sequences and evacuation were later analyzed on cine mode on Philips workstation.

\section{Images analysis:}

The anal sphincter complex was evaluated for the presence of sphincteric defects, scarring, as well as assessment of its relative bulk. Puborectalis and Iliococcygeus muscle slings were assessed for their symmetry, preserved bulk and attachment to the symphysis pubis and obturator internus muscle respectively, where the former was evaluated in the axial plane and latter in the coronal plane.

The Pubococcygeal Line (PCL) was the main reference line to assess the position of the anorectal junction, urinary bladder base and uterus in the midsagittal plane at rest and their descent during evacuation.

Measurement of pelvic floor descent was based on the Rule of 3 to suggest the degree of severity: Descent by $3 \mathrm{~cm}$ or less below the PCL was considered mild, between 3 and 6 was considered moderate and more than $6 \mathrm{~cm}$ was considered severe. 


\section{Results}

A total of 30 patients were examined with a mean age of 40.2. Out of the 30 examined patients $20(66.7 \%)$ were females and $10(33.3 \%)$ were males. All the 30 patients tolerated the examination and were compliant during the dynamic scans with sufficient images, yet the evacuation phase was repeated more than once in $8(26.7 \%)$ patients until the injected gel was seen passing through the anal lumen.

Dynamic MRD revealed rectocele in $19(63.3 \%)$ of all patients, 17 of them were females and only two males (Table 1).

Cystocele was present in $17(56.7 \%)$ patients all of them were females. Four patients (13.3\%) had rectal intussusception; again all of them were females. Anismus was found in 2 males (6.75\%). Enterocele was found in only one (3.3\%) female patient.

Degree of anorectal junction descent below the PCL graded according to the Rule of 3 mild $(<3$ $\mathrm{cm})$ [3 patients $(10 \%)]$, moderate $(3-6 \mathrm{~cm})$ [26 patients $(86.7 \%)]$ and severe $(>6 \mathrm{~cm})(1$ patient $3.3 \%)$.

Multicompartmental dysfunction was observed in 18 female patients $(60 \%)$. None of the patients showed anal sphincter, puborectalis or iliococcygeus defects or scarring with overall rather preserved bulk.

\section{Statistical analysis:}

Statistical analysis was done with SPSS, statistical package (SPSS, Version 17.0).

Table (1): Analysis of the presence and diameters of rectoceles in the evacuation phase classified according to gender.

\begin{tabular}{llll}
\hline & $\begin{array}{c}\text { Female } \\
(\mathrm{n}=20)\end{array}$ & $\begin{array}{c}\text { Male } \\
(\mathrm{n}=10)\end{array}$ & $p$-value \\
\hline No rectocele & $3(15 \%)$ & $8(80 \%)$ & 0.001 \\
Mild $(<2 \mathrm{~cm})$ & $0(0 \%)$ & $1(10 \%)$ & \\
Moderate $(2-4 \mathrm{~cm})$ & $16(80 \%)$ & $1(10 \%)$ & \\
Severe $(>4 \mathrm{~cm})$ & $1(5 \%)$ & $0(0 \%)$ & \\
\hline
\end{tabular}

Table (2): Analysis of the anorectal junction descent in the evacuation phase classified according to gender.

\begin{tabular}{lccc}
\hline $\begin{array}{l}\text { ARJ } \\
\text { descent }\end{array}$ & $\begin{array}{c}\text { Female } \\
(\mathrm{n}=20)\end{array}$ & $\begin{array}{c}\text { Male } \\
(\mathrm{n}=10)\end{array}$ & \multicolumn{1}{c}{$\begin{array}{c}\text { Total } \\
(30)\end{array}$} \\
\hline Mild & 1 & 2 & $3(10 \%)$ \\
Moderate & 18 & 8 & $26(86.7 \%)$ \\
Severe & 1 & 0 & $1(3.3 \%)$ \\
\hline
\end{tabular}

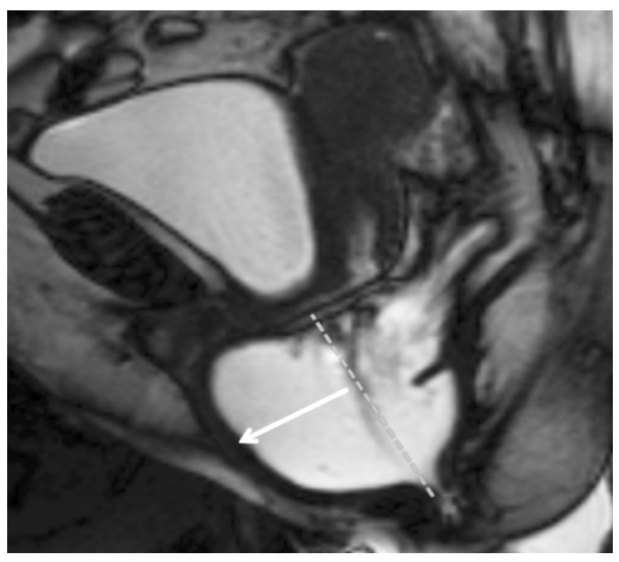

Fig. (1): Anterior rectocele; mid sagittal a line is drawn along the anterior limit of the anal canal (red dashed line) and a line is drawn perpendicular to it do its exact measurement (yellow arrow).

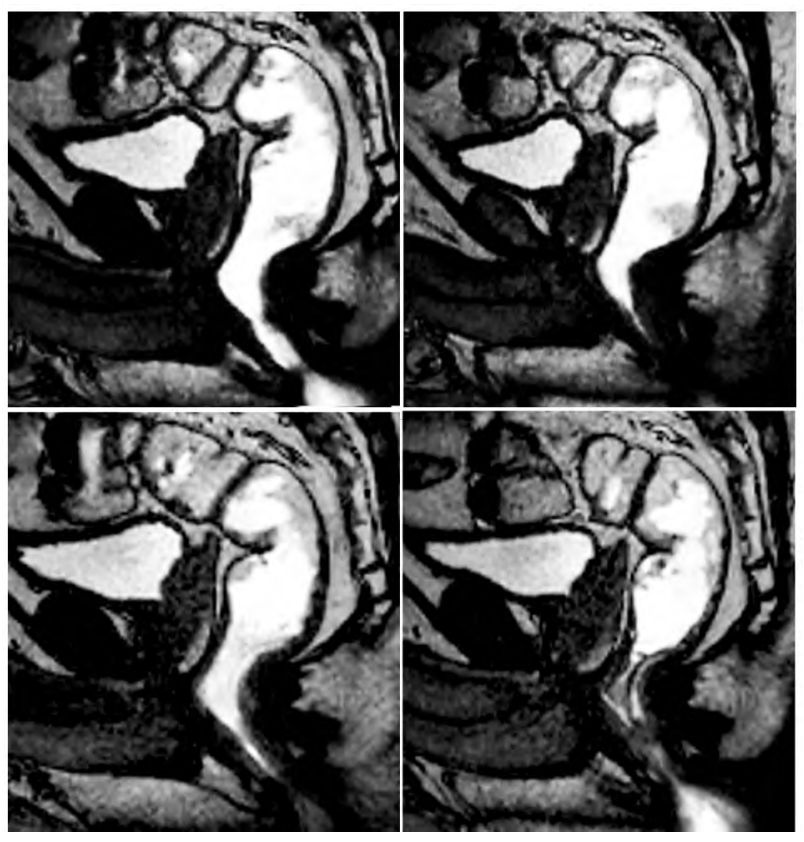

Fig. (2): 45-year old male patient complained of ODS, MRD confirmed the clinical suspicion of anismus due to lack of relaxation of the puborectalis muscle with almost no change in the anorectal angle configuration.

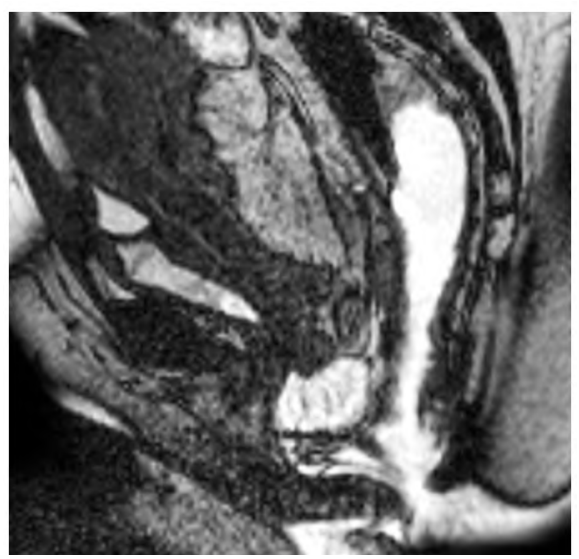

Fig. (3): 36 year old female complaining of ODS, MRD revealed an enterocele with small anterior rectocele and recto-anal intussusception. 


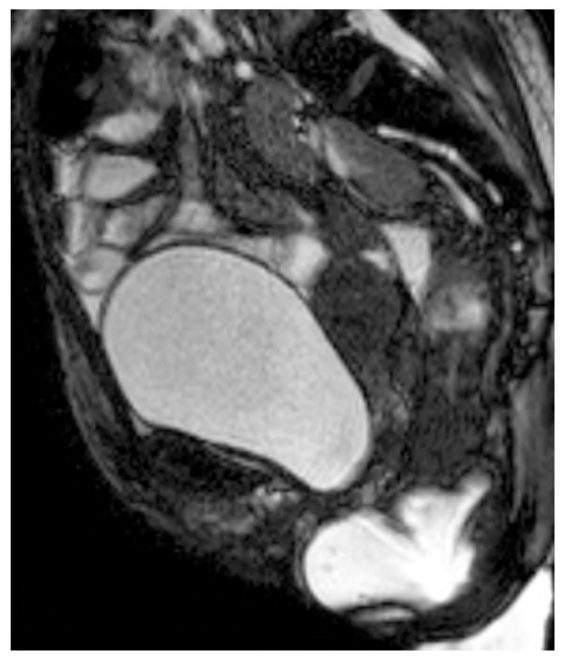

Fig. (4): 28 year old female with obstructed defecation symptoms colonoscopy showed solitary rectal ulcer confirmed by histopathology, MRD Revealed global pelvic floor weakness showing pelvic organ prolapse evident by small cystocele, mild uterine descent and large anterior rectocele with full thickness rectorectal intussusception.

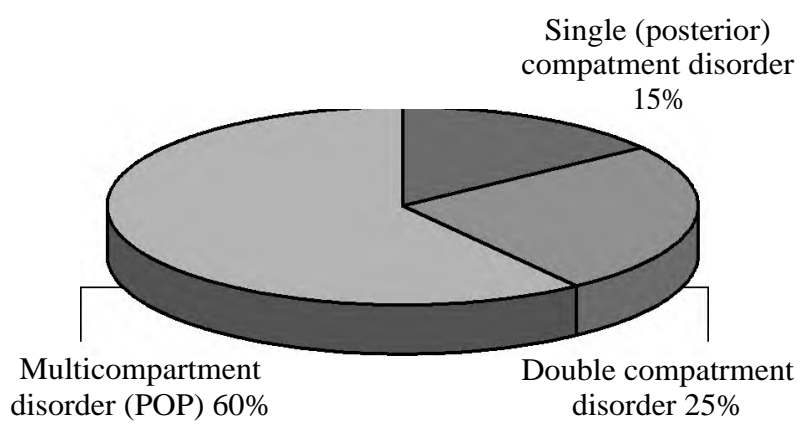

Fig. (5): Pie chart showing the distribution of female patients with ODS according their MR defecography findings into single compartment (posterior), double compartment (posterior + anterior/middle) or multicompartmental (pelvic organ prolapse or POP) disorder.

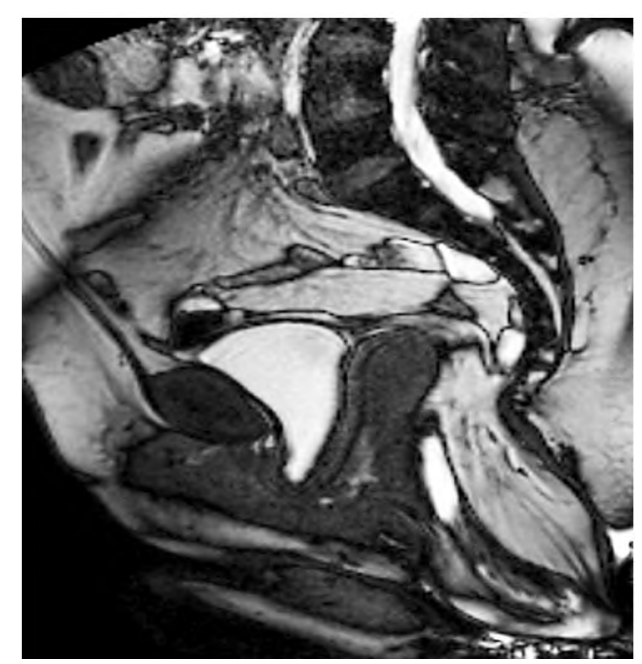

Fig. (6): Global pelvic floor weakness evident by involvement of all three compartments with cystocele, uterine descent and complete external rectal prolapse.

\section{Discussion}

We prospectively studied 30 patients with a provisional diagnosis of obstructed defecation symptoms referred to us at the Radiology Department. Static and dynamic MR defecography examination was performed using a $1.5 \mathrm{~T}$ Philips Acheiva closed configuration machine with a phased array coil.

Multiplanar T2-weighted images were acquired to assess the pelvic floor muscles statically as well as the anal sphincter complex to review anatomical landmarks prior to the dynamic scan.

Dynamic MRD was done using Balanced Fast Field Echo sequences (BFFE) at different grades of straining and during evacuation. Images were later looped on cine mode to visualize the underlying dysfunction ideally.

The midsagittal plane was our corner stone to perform all the relevant measurements using the PCL as our main reference line which is defined as the line connecting the inferior surface of the pubic symphysis and the lowest tip of the coccyx.

Rectocele defined as rectal wall bulging whether anterior or less commonly posterior was found in 17 females and two males Fig. (1). This was in agreement with Elshazly et al., 2010 who stated that rectoceles are diagnosed more frequently in women than in men because the recto-vaginal septum is a relatively weak structure [9].

Two male patients were found to have abnormal puborectalis muscle contraction during the evacuation phase (anismus) as their anorectal angles showed almost no change in configuration on the evacuation phase, rendering the diagnosis of anismus definite Fig. (2). They were treated with biofeedback and physiotherapy and/or Botulinum toxin injection.

Four female patients were found to have low recto-rectal to recto-anal intussusception which combined with the presence of anterior rectocele changes the surgical approach to stapled transanal rectal resection (the STARR procedure) Figs. $(3,4)$.

Cystocele which is defined as the descent of urinary bladder below the PCL which is also graded according to the Rule of 3 is known to widen the levator hiatus hence further obstructing the posterior compartment leading to the sense of outlet obstruction during defecation. We found cystocele in 17 $(57.6 \%)$ patients where all of them were females. 
For the anorectal junction descent during the evacuation phase mild descent was found in 3 patients $(10 \%)$, moderate descent in 26 patients $(86.7 \%)$ and severe only in one patient $(3.3 \%)$. Observing that $90 \%$ of females and $80 \%$ of males with ODS had moderate descent below the PCL denoting posterior compartment dysfunction.

Out of the twenty ladies who underwent the examination, MRD revealed posterior compartment involvement only in $3(15 \%)$ patients and $5(25 \%)$ patients showed concomitant dysfunction in the anterior or middle compartments, while $12(60 \%)$ patients global pelvic floor dysfunction with concomitant dysfunctions of the anterior and middle compartments in addition to the posterior compartment disorders Figs. $(5,6)$.

\section{Conclusion:}

MRD has the ability in diagnosing subtle structural and functional abnormalities that could be overlooked during clinical examination and conventional defecography studies shaping up to be a reliable method of functional imaging of the pelvic floor dysfunction in general and anorectal dysfunction in particular.

\section{References}

1- COLAIACOMO M.C., MASSELLI G., POLETTINI E., LANCIOTTI S., CASCIANI E., BERTINI L. and GUALDI G.: Dynamic MR imaging of the pelvic floor: A pictorial review. Radiographics: A Review Publication of the Radiological Society of North. America., Inc., 29, e35, 2009.
2- National Institute for Health and Clinical Excellence. Stapled Transanal Rectal Resection for Obstructed Defecation Syndrome. NICE Interventional Procedure Guidance (IPG 351) Issued June, Available from: http:// . www.nice.org.uk., 2010

3- EL-SAYED R.F.: The urogynecological side of pelvic floor MRI: The clinician's needs and the radiologist's role. Abdominal Imaging, 38 (5): 912-29, 2013.

4- PODZEMNY V., PESCATORI L.C. and PESCATORI M.: Management of obstructed defecation., 21 (4): 105360, 2015.

5- PILONI V., TOSI P. and VERNELLI M.: MR-defecography in Obstructed Defecation Syndrome (ODS): Technique, diagnostic criteria and grading. Techniques in Coloproctology., 17 (5): 501-10, 2013.

6- EL-SAYED R.F., EL MASHED S., FARAG A., MORSY M.M. and ABDEL-AZIM M.S.: Pelvic floor dysfunction: Assessment with combined analysis of static and dynamic MR imaging findings. Radiology, 248 (2): 518-30, 2008.

7- GARCÍA DEL SALTO L., De MIGUEL CRIADO J., AGUILERA DEL HOYO L.F., GUTIÉRREZ VELASCO L., FRAGA RIVAS P., MANZANO PARADELA M. and FRAILE MORENO E.: MR imaging-based assessment of the female pelvic floor. Radiographics: A Review Publication of the Radiological. Society of North. America., Inc., 34 (5): 1417-39, 2014.

8- EL-SAYED R.F., ALT C.D., MACCIONI F., MEISSNITZER M., MASSELLI G., MANGANARO L. and WEISHAUPT D.: Magnetic resonance imaging of pelvic floor dysfunction-joint recommendations of the ESUR and ESGAR Pelvic Floor Working Group. European. Radiology, 27 (5): 2067-85, 2017.

9- ELSHAZLY W.G., EL-NEKADY A.E. AZEZ and HASSAN H.: Role of dynamic magnetic resonance imaging in management of obstructed defecation case series. International Journal of Surgery, 8 (4): 274-82, 2010.
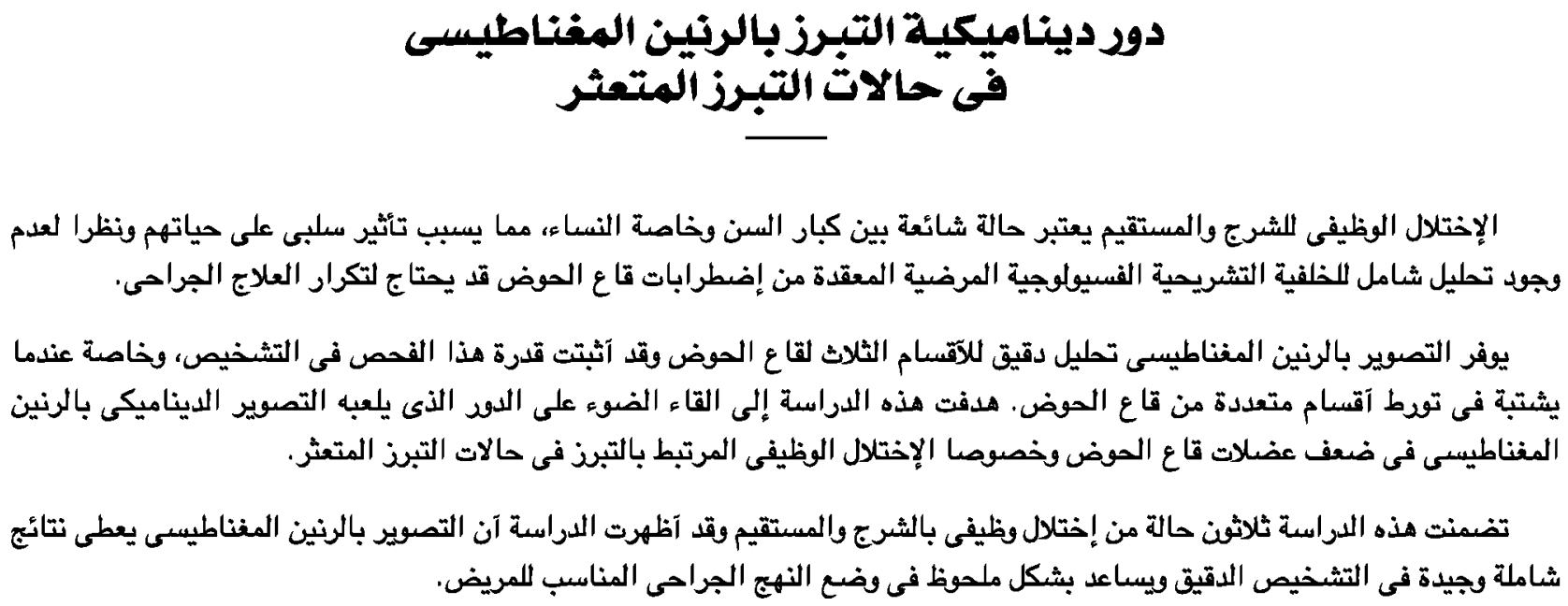